ARTÍCULO ORIGINAL

\title{
SISTEMAS DE INTERPRETACIÓN, ITINERARIOS Y REPERTORIOS TERAPÉUTICOS DE PACIENTES CON LEPRA EN UN PAIIS CON BAJA PREVALENCIA
}

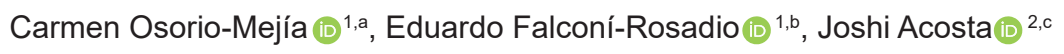 \\ 1 Instituto Nacional de Salud, Lima, Perú. \\ 2 Instituto de Evaluación de Tecnologías en Salud e Investigación, ESSALUD, Lima, Perú. \\ a Bióloga microbióloga salubrista; ${ }^{\mathrm{b}}$ medico infectólogo y medicina tropical; ' médica microbiologa, doctora en \\ Microbiología.
}

\section{RESUMEN}

Objetivos: En Perú, a pesar del escaso número de casos, existe evidencia de un diagnóstico tardío y prevalencia oculta de la lepra. En este contexto el objetivo del estudio fue conocer los sistemas de interpretación sobre la lepra, itinerarios y repertorios terapéuticos de pacientes con diagnóstico de lepra que se encuentren en tratamiento o con tratamiento culminado. Materiales y métodos: Se realizó un estudio cualitativo, aplicando entrevistas semiestructuradas a pacientes con diagnóstico de lepra de las regiones de Loreto y Ucayali. Resultados: Se entrevistaron a 30 pacientes. La mayoría no conocía el mecanismo de transmisión de la lepra. En relación con los itinerarios terapéuticos, los pacientes generalmente acudieron a los establecimientos de salud por recomendación de terceros que conocían la enfermedad. En algunos casos, el personal de salud realizó un mal diagnóstico. Se reconoce la importancia del tratamiento indicado por el Ministerio de Salud; sin embargo, factores económicos y la distancia a los establecimientos de salud afectan de forma negativa la adherencia al tratamiento. Además, se evidenció que persiste el estigma de la enfermedad. Conclusiones: Los pacientes reconocen la importancia del tratamiento, sin embargo, manifiestan ideas equivocadas sobre la patogenia de la lepra, además se identifican debilidades en el sistema de salud. Estos problemas conllevarían al retraso en el diagnóstico y tratamiento. Se recomienda fortalecer las estrategias de control y descentralizar la atención de la lepra con la participación de la comunidad, pacientes, personal de salud y curanderos, considerando las barreras identificadas y un probable infradiagnóstico en la mujer.

Palabras claves: Lepra; Servicios de Salud; Percepción; Tratamiento; Investigación Cualitativa; Discapacidad; Factores Sociodemográficos; Estigma; Perú (fuente: DeCS BIREME).

\section{INTERPRETATION SYSTEMS, THERAPEUTICITINERARIES AND REPERTOIRES OF LEPROSY PATIENTS IN A LOW PREVALENCE COUNTRY}

Citar como: Osorio-Mejía C, Falconí-Rosadio E, Acosta J. Sistemas de interpretación, itinerarios y repertorios terapéuticos de pacientes con lepra en un país con baja prevalencia. Rev Peru Med Exp Salud Publica. 2020;37(1):2531. Doi: https://doi.org/10.17843/ rpmesp. 2020.371 .4820

Correspondencia: Carmen Edith Osorio Mejía; Av. Defensores del Morro 2268, Chorrillos. Lima, Perú; caome22@gmail.com

\section{ABSTRACT}

Objectives: In Peru, despite the small number of cases, there is evidence of late diagnosis and hidden prevalence of leprosy. In this context the objective of the study was to know the interpretation systems on leprosy, itineraries and therapeutic repertoires of patients diagnosed with leprosy who are in treatment or who have finished treatment. Materials and methods: A qualitative study was carried out, applying semi-structured interviews to patients diagnosed with leprosy from the Loreto and Ucayali regions. Results: 30 patients were interviewed. Most did not know the mechanism of leprosy transmission. In relation to therapeutic itineraries, patients generally went to health facilities on the recommendation of third parties who knew the disease. In some cases, health personnel made a bad diagnosis. The importance of the treatment indicated by the "Ministerio de Salud" (Ministry of Health) is recognized; however, economic factors and the distance to health facilities negatively affect adherence to treatment. In addition, it was evidenced that stigma persists towards the disease. Conclusions: Patients recognize the importance of treatment; however, they express misconceptions about the pathogenesis of leprosy, and weaknesses in the health system are also identified. These problems would lead to delay in diagnosis and treatment. It is recommended to strengthen control strategies and decentralize the care of leprosy with the participation of the community, patients, health personnel and healers, considering the identified barriers and a probable underdiagnosis in women.

Keywords: Leprosy; Health services; Perception; Therapeutics; Qualitative Research; Disability; Sociodemographic Factors; Stigma; Perú (source: MeSH NLM).
Recibido: 20/09/2019

Aprobado: $22 / 01 / 2020$

En línea: 19/03/2020 


\section{INTRODUCCIÓN}

En el 2000, se reportó la eliminación de la lepra como problema de salud pública a nivel mundial, y en el 2005 la mayoría de países habían logrado esta meta; sin embargo, en muchos países continúa siendo un problema a nivel subnacional ${ }^{(1)}$.

En Perú, según información brindada por el Ministerio de Salud (MINSA), se observa una disminución progresiva de la prevalencia, aunque todavía hay detección de nuevos casos. A pesar de ello, se ha logrado alcanzar tasas menores a 1 por 10000 habitantes a nivel subnacional (provincias).

La mayoría de los casos se circunscriben en la selva peruana, específicamente en las regiones Loreto y Ucayali, debido a que la enfermedad ingresó a estas regiones a través de la frontera con Brasil en el siglo XX ${ }^{(2)}$. En este contexto, el gobierno planteó políticas para el control de la lepra y facilitó la introducción de medicamentos antileprosos, lográndose el control de la enfermedad, pero no su erradicación ${ }^{(2)}$. En 2017, el MINSA reportó once nuevos casos, incluyendo algunos con discapacidad grado II, que evidencia un diagnóstico tardío y una prevalencia oculta de la enfermedad.

El infradiagnóstico y diagnóstico tardío se relacionan con la falta de actividades de seguimiento y la búsqueda activa de casos, y a la desinformación de la población y del personal sanitario, influenciando de forma negativa en el control de la lepra ${ }^{(3)}$.

Además, el diagnóstico tardío está asociado con la demora en la búsqueda de atención sanitaria por problemas económi$\cos ^{(4)}$, y con el itinerario terapéutico que siguen los pacientes, entendido como lo que hacen las personas y a quienes acuden para mantener o restablecer su salud ${ }^{(5)}$. También se deben considerar el impacto de la medicina tradicional, que presenta diferentes concepciones en lo referente a la salud y que abarca experiencias personales; y la cooperación de la familia y la comunidad. ${ }^{(6)}$ La búsqueda de tratamiento, en contextos en que coexisten sistemas de salud diferentes, está influenciada por la complejidad, costos y proximidad sociocultural y geográfica de la oferta, que conlleva a que los pacientes puedan combinar las diferentes ofertas de cada sistema de salud ${ }^{(7,8,9)}$.

El control de la lepra requiere, además, conocer los repertorios terapéuticos (procedimientos y recursos) existentes en cada sistema de salud y cómo son utilizados por los pacientes ${ }^{(10)}$. Asimismo, se necesita de una adecuada adherencia al tratamiento; sin embargo, esta se ve influenciada por factores socioeconómicos, culturales, psicosociales, conductuales y por las reacciones adversas a los fármacos ${ }^{(11,12)}$. En este contexto, se debe considerar que los sistemas de interpretación de la enfermedad varían dentro y entre grupos culturales, influenciando en la forma como las personas actúan para prevenir y tratar la enfermedad ${ }^{(6,13-17)}$. Considerando esto, el objetivo del estudio fue conocer los sistemas de interpretación sobre la lepra, itinerarios y repertorios terapéuticos de pacientes con diagnóstico de lepra que se encuentren en tratamiento o con tratamiento culminado.

\section{MENSAJES CLAVE}

Motivación para realizar el estudio: En Perú, un país con baja prevalencia de lepra, aún se diagnostican pacientes con discapacidad grado II por lepra, que sugiere un diagnóstico tardío de la enfermedad.

Principales hallazgos: Los pacientes no conocen el mecanismo de transmisión de la lepra, la mayoría acude al hospital por recomendación de terceros que identificaron la enfermedad. Existen factores económicos y de accesibilidad que afectan la adherencia al tratamiento.

Implicancias: Se requieren estrategias para controlar de la lepra, considerando las limitaciones identificadas del sector salud y el desconocimiento de los pacientes sobre la enfermedad.

\section{MATERIALES Y MÉTODOS}

Se realizó un estudio cualitativo, utilizando un muestreo no probabilístico intencional, en el cual se aplicó entrevistas a profundidad semiestructuradas a pacientes con diagnóstico de lepra que se encontraban en tratamiento o con tratamiento culminado. Las entrevistas se realizaron entre octubre y noviembre del 2015. Previamente, el entrevistador y las responsables de la Estrategia Sanitaria Nacional de Tuberculosis Componente Lepra tuvieron una reunión con los pacientes con el fin de explicarles de manera sencilla y clara el propósito de la investigación.

Se incluyeron pacientes mayores de 18 años con diagnóstico de lepra realizado en los establecimientos de salud del MINSA, que residían, al momento del estudio, en la ciudad de Iquitos, Loreto (provincia de Maynas, distritos de Belén, Punchana y San Juan Bautista); y en la ciudad de Pucallpa, Ucayali (provincia de Coronel Portillo, distritos de Callería, Yarinacocha y Manantay). Se establecieron ocho perfiles para los pacientes en cada región considerando: tratamiento de lepra (en tratamiento y tratamiento culminado), domicilio (urbano y rural) y sexo (femenino y masculino).

Las entrevistas fueron realizadas por un profesional de salud con experiencia en trabajos comunitarios en diversas comunidades nativas y en la realización de estudios cualitativos. Previamente tuvo una inducción respecto al tema de investigación por parte del equipo de investigadores (antropólogo e infectólogo experto en el manejo de pacientes con lepra). El entrevistador además vivió en una de las regiones incluidas, lo que facilitó la comunicación con los pacientes, ya que conocía las costumbres y los términos culturales con los cuales se comunicaban coloquialmente. Cabe mencionar que todas las entrevistas fueron realizadas en las casas de los pacientes, asegurando la privacidad y la confidencialidad.

Considerando las teorías de Chrisman ${ }^{(5)}$,Kleinman ${ }^{(6)}$, Young ${ }^{(7)}$, Schartz ${ }^{(8)}$ y Good ${ }^{(13)}$, se plantearon tres dimen- 
siones de estudio: sistemas de interpretación sobre la lepra, itinerarios terapéuticos y repertorios terapéuticos que utilizan los pacientes con diagnóstico de lepra, en esta última dimensión se incluyó la adherencia al tratamiento. Luego para cada dimensión se plantearon códigos para el análisis (20 en total). En base al objetivo y las dimensiones de estudio. Ademas, se preparó la guía de entrevista, la cual fue revisada por un dermatólogo experto en lepra y un salubrista con experiencia en trabajos comunitarios, y modificada de acuerdo con sus sugerencias. La versión final de la guía fue aplicada a las personas que decidieron voluntariamente participar en el estudio. Las entrevistas se realizaron hasta alcanzar el punto de saturación de la información, lográndose obtener un total de 30 entrevistas.

Se planteó un análisis inductivo, para lo cual se procedió a la transcripción de las entrevistas, y se realizó un análisis de contenido de cada una de ellas, en Excel, encontrándose información solo de 16 de los 20 códigos planteados inicialmente. Durante este análisis se encontró una dimensión de análisis adicional: impacto de la lepra en la vida de las personas, pero que no fue considerada por no ser objeto del estudio. Los 16 códigos de análisis fueron validados por dos especialistas en el tema (coordinador del programa de la lucha contra la lepra a nivel nacional y un dermatólogo del Hospital Regional de Iquitos). Se realizó un análisis descriptivo de las características sociodemográficas de los sujetos de estudio, resumiendo las variables numéricas según su mediana y las variables categóricas según su porcentaje. Posteriormente se realizaron viajes a Loreto y Ucayali para realizar la triangulación de los datos y obtener la retroalimentación de los participantes acerca de los hallazgos.

El presente estudio fue aprobado por el Comité de ética del Instituto Nacional de Salud del Perú mediante Memorando 080-2015-CIEI-INS (Código OI-052-14). Además, a los pacientes que aceptaron participar en el estudio, se les solicitó firmar el consentimiento informado.

\section{RESULTADOS}

Se incluyeron a 30 pacientes, de los cuales 10 eran mujeres, 10 eran procedentes del área rural y 20 habían culminado su tratamiento. La mayoría de los pacientes que culminaron el tratamiento eran mayores de 50 años y recibieron su primer tratamiento en el leprosario de San Pablo (Tabla 1).

Se realizaron entrevistas para los siguientes perfiles: Perfil en tratamiento, urbano, masculino (7 pacientes), perfil en tratamiento, urbano, femenino (1 paciente), perfil en tratamiento rural, masculino ( 2 pacientes), perfil en tratamiento culminado, urbano, masculino (7 pacientes), perfil en tratamiento culminado, urbano, femenino (5 pacientes), perfil en tratamiento culminado, rural, masculino (4 pacientes) y perfil en tratamiento culminado, rural, femenino (4 pacientes). Se presenta el análisis por dimensiones, considerando los códi- gos de los hallazgos más relevantes. El análisis completo se adjunta como material suplementario: anexo 1, 2 y 3 .

\section{Sistemas de interpretación sobre la lepra}

Independientemente del sexo, edad y zona de procedencia del paciente, se identificaron diversas respuestas en lo referente a la forma de contagio de la lepra: presencia de alguna persona extraña o de un enfermo en casa, lavar la ropa de un enfermo o darle la mano, contacto sexual con un enfermo, haberse desplazado fuera de sus comunidades, exposición a agentes contaminantes como aguas servidas o enfermedades del bosque, o la ingesta de ciertos alimentos (como el tapir amazónico o el armadillo), la voluntad divina y factores hereditarios.

«Pero de repente mis antepasados han tenido, pero tú sabes el orden genético, nunca desaparece, sigue su ritmo de repente tus hijos no tienen, pero tus tataranietos si pueden tener ...»

Hombre, 55 años, en tratamiento, área urbana, Ucayali.

Otra percepción fue que los microorganismos causantes de la lepra u otras enfermedades están con la persona desde que nace y que esta se desarrolla dependiendo de la respuesta inmune.

La transmisión por vía aérea también fue mencionada por tres pacientes, quienes refirieron que la lepra se contrae al conversar con una persona enferma; sin embargo, ellos no muestran seguridad en esta respuesta, ya que posteriormente justifican su contagio por otras modalidades. También se logró identificar que la percepción de que la lepra es una enfermedad altamente contagiosa aún está presente (hombre de zona rural, 46 años); mientras que, otros entrevistados refirieron que no era contagioso (mujer de zona rural, 76 años y mujer de zona urbana, 78 años).

Los pacientes, independientemente de la zona de procedencia y sexo, de edades entre 70 y 79 años, refirieron cuatro formas de prevención para evitar contagiar la enfermedad: no tener relaciones sexuales con el afectado, dormir separados, no besar en la boca, quemar colchones y sábanas después de curarse, tener los útiles personales separados y alimentarse bien.

«iUsted mismo ha dicho mejor duermo a parte o tu esposa te ha dicho? Yo mismo he dicho mejor dormiré aparte porque de repente la contagio...»

Hombre, 41 años, en tratamiento, área urbana, Ucayali.

Con relación a las consecuencias del abandono del tratamiento, las respuestas fueron: recaídas, muerte, discapacidad y muerte por complicaciones. Este discurso lo tuvieron todos los pacientes independientemente de la zona de procedencia, sexo y edad.

«Si abandona el tratamiento, le puede pasar otra vez, (...) de repente no toma pues, tiene que caer de nuevo, puede suceder la muerte también».

Mujer, 71 años, curada, área rural, Loreto. 
Tabla 1. Características sociodemográficas de los pacientes incluidos en el estudio

\begin{tabular}{|c|c|c|c|c|}
\hline \multirow{2}{*}{ Variable } & \multicolumn{2}{|c|}{ Loreto } & \multicolumn{2}{|c|}{ Ucayali } \\
\hline & $n=16$ & $\%$ & $n=14$ & $\%$ \\
\hline \multicolumn{5}{|l|}{ Sexo } \\
\hline Hombre & 9 & 56,3 & 11 & 78,6 \\
\hline Mujer & 7 & 43,7 & 3 & 21,4 \\
\hline \multicolumn{5}{|l|}{ Procedencia } \\
\hline Urbana & 11 & 68,7 & 10 & 71,4 \\
\hline Rural & 5 & 31,3 & 4 & 28,6 \\
\hline \multicolumn{5}{|l|}{ Estado civil } \\
\hline Casada & 6 & 37,5 & 3 & 21,4 \\
\hline Soltera & 2 & 12,5 & 4 & 28,6 \\
\hline Divorciada & 1 & 6,3 & 3 & 21,4 \\
\hline Conviviente & 5 & 31,2 & 4 & 28,6 \\
\hline Viudo/a & 2 & 12,5 & 0 & 0,0 \\
\hline \multicolumn{5}{|l|}{ Condición de tratamiento } \\
\hline $\begin{array}{l}\text { Con tratamiento culmi- } \\
\text { nado }\end{array}$ & 12 & 75,0 & 8 & 57,1 \\
\hline En tratamiento & 4 & 25,0 & 6 & 42,9 \\
\hline Edad (años), mediana (RIC) & \multicolumn{2}{|c|}{$60,5(23,2)$} & \multicolumn{2}{|c|}{$56(27,7)$} \\
\hline $\begin{array}{l}\text { Número de miembros de la } \\
\text { familia que viven en casa, } \\
\text { mediana (RIC) }\end{array}$ & \multicolumn{2}{|c|}{$5(2)$} & \multicolumn{2}{|c|}{ No hay dato } \\
\hline $\begin{array}{l}\text { Tiempo de duración de las } \\
\text { entrevistas, mediana (RIC) }\end{array}$ & \multicolumn{2}{|c|}{$40(31)$} & \multicolumn{2}{|c|}{$61(26,2)$} \\
\hline
\end{tabular}

RIC: rango intercuartil

Usualmente se maneja el discurso de que la lepra es curable siguiendo la poliquimioterapia; sin embargo, una paciente de zona rural de 81 años mencionó que era incurable ya que la enfermedad está permanentemente en la sangre. Por otro lado, se reconoce que antes no había tratamiento para la lepra y que los pacientes se morían.

«Bueno me ha dicho que es una enfermedad que sí se puede curar, no es una enfermedad incurable, hoy en la actualidad tiene cura, muchas personas (...) he conversado con el doctor entonces donde él me ha indicado que es lo que debo hacer»

Hombre, 58 años, en tratamiento, área urbana, Loreto.

\section{Itinerarios terapéuticos en la lepra}

Independientemente del sexo y la edad, los pacientes destacaron que el apoyo de su familia (especialmente la esposa), es importante para que acudan al establecimiento de salud, y posteriormente cumplan el tratamiento y los controles. Sin embargo, una mujer de la zona rural de 71 años, manifestó que sus familiares le dieron la espalda por temor al contagio. Otro paciente, manifestó que su profesor identificó las manchas y lo llevó al hospital para el diagnóstico.

Un mal diagnóstico por la falta de experiencia del personal de salud, puede ocasionar que el paciente pierda la confianza y busque otras opciones de tratamiento, pudiendo conllevar al retraso del diagnóstico. Se identificó este hallazgo en dos pacientes jóvenes menores de 35 años de la zona urbana.
Tres pacientes menores de 50 años (dos de zona rural y uno de zona urbana) con tratamiento culminado refirieron haber consultado a un curandero al no encontrar mejoría de los síntomas. Un paciente varón de 26 años de la zona urbana mencionó que el curandero lo refirió al hospital. Manifestó que no creía en brujos, pero que fue llevado por sus hermanas.

«¿Usted fue al curandero? Sí, de aquellos que curan hechicerías (...) fui porque mis hermanas me recomendaron al verme que seguía igual. Fui después del tratamiento y yo pensaba que cuando termine el tratamiento, ya se terminó todo, pero no es así en realidad, demora en desaparecer de tu cuerpo».

Hombre, 26 años, en tratamiento, área urbana, Ucayali.

Independientemente de la zona de procedencia y el sexo, los pacientes refieren que las ofertas de salud para la atención de la lepra son limitadas, de difícil accesibilidad geográfica y con falta de especialistas, lo que constituyen barreras para acceder al diagnóstico y al tratamiento temprano.

Se identificó al hospital y al programa de lepra como el lugar donde primordialmente se trata la enfermedad. Los pacientes también señalaron otros lugares de atención, como el Hospital de la Solidaridad en Lima, el Instituto de Medicina Tropical de la Universidad Nacional Mayor de San Marcos y clínicas privadas. En los entrevistados mayores de 70 años que culminaron su tratamiento, se identificó como el lugar de atención al leprosario de San Pablo. Tampoco fueron reconocidas como opciones las boticas ni las farmacias.

«Se ha hecho ronchas (...) la gente más hablaba, y como en Pucallpa también había enfermos que han venido en balsa, atrás en Contamana, me recogieron y llevaron a San Pablo» Mujer, 67 años, curada, área rural, Loreto.

Asimismo, la mitad de los entrevistados identificó a un determinado médico o técnico del MINSA, como la persona que realiza el diagnóstico o brinda tratamiento (se identificó un solo médico o técnico para cada una de las ciudades de donde provenían los pacientes).

\section{Repertorio terapéutico de la lepra y adherencia al tratamiento}

En relación a los tratamientos utilizados, los pacientes indicaron que tomaron diferentes tipos de fármacos a lo largo de los años, y además, usaron vegetales, barro y plantas medicinales, como la ayahuasca, chirimasango con sacha ajo, catahua, marco sacha, corteza de cedro, etc. Estos tratamientos eran dados por familiares o curanderos.

«... y en esa época todavía mi papá vivía y me dijo: ya te voy a traer un remedio que es efectivo», y me trajo el sacha ajoma macho, este es una raíz selvática (...). Y después el chirisamango. He tomado un litro y desapareció ese adormecimiento más o menos como diez años»

Hombre, 58 años, en tratamiento, área urbana, Loreto.

Los pacientes manifestaron la importancia de iniciar el tratamiento en forma precoz como una medida para evitar 
el contagio o las complicaciones, pero también se evidenció que los pacientes tienen la percepción que, a pesar de haber completado el tratamiento, la lepra podía estar dentro de ellos y podría volver a manifestarse.

Con relación a los factores externos que afectan en forma negativamente la adherencia al tratamiento tenemos el vivir lejos de los centros de salud, limitado horario de atención de los centros de salud y recursos económicos limitados especialmente en los pacientes de zona rurales. También fueron mencionadas las reacciones secundarias (especialmente gastrointestinales), estilo de vida del paciente (beber y fumar) y la presencia de otra enfermedad, como por ejemplo los problemas articulares a nivel de rodilla, pues manifiestan que no pueden tomar tanta medicina al mismo tiempo (hombre de zona urbana, 70 años).

«No, no me ido porque yo prácticamente yo vivía acá no tenía recursos para salir para irme a Atalaya, a Pucallpa, (...) por falta de recursos pues»

Hombre, 49 años, en tratamiento, área rural, Ucayali.

Aproximadamente la mitad de los pacientes manifiestan tener Seguro Integral de Salud (SIS), facilidades de parte de los médicos para atenderlos y transferencias a establecimientos de salud más cercanos a su pueblo para recibir el tratamiento. Independientemente del sexo, zona de procedencia y edad se identificó que el acompañamiento de la familia, especialmente la esposa e hijos, favorece la adherencia. No se logró identificar que los amigos o vecinos cumplieran este rol.

Se identificaron pacientes, independientemente de la zona de procedencia, sexo y edad que refieren evitar consumir carnes rojas, o alimentos ricos en grasas, algunos pescados, ají o beber cerveza durante el tratamiento con la finalidad de no incrementar las alteraciones hepáticas o renales asociadas al tratamiento. Evitan, también, el consumo de pescado con espinas o cerdo porque lo relacionan con la aparición de lesiones lepromatosas.

«¿Y cuándo usted estaba en tratamiento qué cosas no comía? Cuando comencé a tragar las pastillas todos los días, pero comía de todo, solo no tomaba cerveza, no fumaba, no comía chancho, ají...»

Hombre, 46 años, con tratamiento culminado, área rural.

\section{DISCUSIÓN}

El presente estudio muestra que la mayoria de los pacientes que estaban recibiendo o habían recibido tratamiento en un establecimiento de salud del MINSA, desconocían el modo de contagio de la lepra. Aunque la mayoría lo asoció a una forma de contagio, solo tres pacientes mencionaron la vía aérea. Esto podría explicarse por la representación social de la enfermedad, asociada a percepciones de incurabilidad y contagiosidad, lo que a su vez se relaciona con el estigma asociado a las deformidades físicas ${ }^{(14,15)}$. La mayoría de los pacientes entrevistados habían culminado el tratamiento, y muchos presentaban secuelas, por inicio tardío del tratamiento, o porque contrajeron la enfermedad hace muchos años cuando no existía tratamiento específico, lo que puede explicar las percepciones de incurabilidad asociadas a falta de entendimiento y conocimiento sobre la enfermedad ${ }^{(15,16)}$. A pesar de que, en nuestro estudio los entrevistados manifiestan que están curados gracias a la poliquimioterapia y reconocen las consecuencias del abandono del tratamiento, esta condición de «curado» desde la percepción del afectado y comunidad es "engañosa», porque algunos pacientes siguen sintiendo que están infectados, tanto entre los que presentan o no discapacidad ${ }^{(15)}$.

El estudio evidenció que la mayoría de las pacientes acudieron a los hospitales o postas por recomendación de terceros. La influencia de los familiares cercanos en la búsqueda de ayuda para el diagnóstico de la enfermedad fue muy importante. La literatura señala que la influencia de la red social motiva a los pacientes a buscar el diagnóstico definitivo; caso contrario, el afectado no lo hace por sí solo; este comportamiento puede deberse a dos motivos: que a pesar de tener una experiencia previa con la enfermedad, estos pacientes no eran conscientes del riesgo al que estaban expuestos, o puede deberse a que, a pesar de sospechar que tienen lepra, callan por temor al rechazo social; por tales motivos, la toma de decisiones acerca de acudir o no a un centro de salud también estaría influenciada por las experiencias sociales negativas acumuladas ${ }^{(18,19)}$.

En relación al itinerario terapéutico, encontramos que la calidad de los servicios de salud, sobre todo del primer nivel y clínicas particulares fue ineficiente. En algunos de los casos dieron un mal diagnóstico, retrasando el inicio del tratamiento; este hallazgo concuerda con Naaz, et al. donde se reporta que los profesionales locales tienen un conocimiento pobre acerca de la lepra, aunque este estudio describe poca derivación a los centros especializados ${ }^{(15)}$. Estos investigadores, han indicado que los pacientes que van a los hospitales de mayor complejidad tienen 6,6 veces más probabilidad de recibir un tratamiento oportuno ${ }^{(15)}$; sin embargo, para nuestra realidad, y en la búsqueda de la erradicación de la lepra, es necesario fortalecer los establecimientos de salud de primer nivel de atención para la captación de probables casos de lepra, mediante tamizaje dermatológico y de los contactos domiciliarios ${ }^{(20)}$.

Es importante resaltar que la mayoría de pacientes refieren que las ofertas de salud son limitadas, que se cuenta con escasos especialista, y en varios casos hay dificultad en el acceso a los establecimientos de salud, ya sea por la lejanía de los establecimientos de salud, o por el costo económico del traslado. Esto constituye barreras para acceder al diagnóstico y tratamiento temprano, y dificultan la adherencia terapéutica.

Además, se ha evidenciado la necesidad de fortalecer el conocimiento sobre la enfermedad en los pacientes, comu- 
nidad y personal de salud, debido a que el desconocimiento de la enfermedad está asociado a retraso en la búsqueda de atención médica y falta de adherencia al tratamiento ${ }^{(21,22)}$. En este sentido, solo dos pacientes señalaron que el inicio del tratamiento es muy importante para evitar la discapacidad, a pesar de que la relación entre discapacidad, tratamiento y diagnóstico oportuno es respaldada por diversos estudios ${ }^{(15,23)}$. El retraso en el diagnóstico, está relacionado al desconocimiento de la enfermedad por parte del personal de salud, por lo tanto, es importante reforzar la educación sobre esta patología en los profesionales de las provincias con casos de lepra ${ }^{(21,22)}$.

Con relación a la búsqueda de atención por curanderos, aproximadamente la tercera parte buscaron atención con curanderos, incluyendo pacientes que se encuentran actualmente en tratamiento. Esto ha sido descrito en otros estudios, donde se encontró que la mayoría de los participantes del estudio recibió el primer tratamiento de brujos y curanderos tradicionales ${ }^{(18)}$. En la actualidad, se ha evidenciado que se confía en los curanderos (aunque en forma limitada), creándose la necesidad de incorporar al curandero como «apoyo médico complementario» dentro del sistema biomédico ${ }^{(24)}$, posiblemente para cumplir roles de mediadores de referencia hacia los hospitales.

El Perú tiene una baja prevalencia de la lepra, por lo tanto, la detección de nuevos casos debería de acompañarse de un estricto seguimiento de los contactos, participación social de los afectados y de la comunidad, y además de profesionales capacitados y centros de vigilancia de resistencia de medicamentos ${ }^{(20,25)}$. Sin embargo, en nuestro estudio y otros previos, se ha evidenciado, dos problemas grandes: la falta de experiencia del personal sanitario en la detección de la enfermedad ${ }^{(15)} \mathrm{y}$ un trabajo desarticulado entre centros de salud, comunidad y hospital especializado, para el control de la lepra. Asimismo, otros estudios han descrito que el estigma y la discriminación son factores psicosociales que juegan un rol importante en la eliminación de la lepra, por lo tanto, las políticas públicas deben estar orientadas a instaurar medidas que aborden estos temas ${ }^{(20,25)}$.

Entre las limitaciones del estudio, tenemos la dificultad para obtener al menos dos entrevistas para cada perfil. Esto se relaciona con la disminución de nuevos casos de lepra en el país, y que los nuevos casos se encuentren dispersos en distritos alejados. Asimismo, se observó que hubo mayor dificultad en acceder a pacientes mujeres en tratamiento tanto de la zona rural como de la urbana, y esto podría limitar nuestros resultados en cuanto a sus percepciones sobre la lepra; sin embargo, esto podría explicarse por la epidemiología de la lepra en el país, donde según los reportes nacionales, los casos nuevos son mayormente en hombres. A pesar de que se ha reportado que el diagnóstico de lepra es más frecuente en hombres que en mujeres, esto difiere estadisticamente de otros países como Brasil, donde los casos de lepra en Mato Grosso son más frecuentes en mujeres, lo cual debe evaluarse con cautela, debido al posible infradiagnóstico de lepra en mujeres ${ }^{(26,27)}$.

Otra limitación importante del estudio fue que la persona que realizó las entrevistas no participó en la concepción del proyecto ni en el análisis de las entrevistas. A pesar de las limitaciones, creemos que los resultados son relevantes, debido a que no se han realizado estudios similares en Perú. Estos resultados pueden ayudar a fortalecer las estrategias para eliminación de la lepra a nivel distrital, considerando aquellas ciudades con casos nuevos en los últimos años.

En conclusión, los pacientes reconocen la importancia del tratamiento de la lepra, sin embargo, manifiestan ideas equivocadas sobre la patogenia de esta enfermedad y se identifican debilidades en el sistema de salud. Estos problemas conllevarían al retraso en el diagnóstico y tratamiento de la enfermedad. Se recomienda fortalecer las estrategias de control y descentralizar la atención de la lepra con la participación de la comunidad, pacientes, personal de salud y curanderos, considerando las barreras identificadas y un probable infradiagnóstico en mujer. De esta forma se incidirá en la erradicación de la lepra en el país.

Agradecimientos: Al Mg. Efraín Ayala Remón, por su apoyo en la realización de las entrevistas y transcripción y al antropólogo Enzo Morales por su apoyo en el análisis de la información.

Contribuciones de autoría: JA y EFR han participado en la concepción y diseño del estudio. JA y COM participaron en la recolección, análisis de los datos y redacción del artículo. Además, JA participó en la obtención de financiamiento. Los tres autores aprobaron la versión final del artículo.

Financiamiento: La investigación fue financiada a través del Fondo Concursable 2014 del Centro Nacional de Salud Pública del Instituto Nacional de Salud de Perú.

Conflictos de interés: Los autores declaran que no tienen ningún conflicto de interés en relación con esta publicación.

Material suplementario: Disponible en la versión electrónica de la RPMESP

\section{REFERENCIAS BIBLIOGRÁFICAS}

1. World Health Organitation. Weekly epidemiological record [Internet] WHO. 2016 [citado el 4 de diciembre del 2019]. Disponible en: https:// www.who.int/wer/2016/wer9135.pdf

2. Burstein Z. Revisión histórica del control de la Lepra en el Perú. Rev perú med exp salud pública [Internet]. 2001 [citado el 28 de diciembre del 2019]; 18(1-2):40-4. Disponible en: http://www.scielo.org.pe/scielo. php?script=sci_arttext\&pid=S1726-46342001000100010\&lng=es
3. Acosta J. Evaluabilidad del Programa de eliminación de la lepra en el Perú [tesis de master]. Granada: Escuela Andaluza de Salud Pública, Universidad de Granada; 2013.

4. PetersRMH,Dadun,LusliM,Miranda-GalarzaB, Van BrakelWH,Zweekhorst $\mathrm{MBM}$, et al. The meaning of leprosy and everyday experiences: an exploration in cirebon, indonesia. J Trop Med [Internet]. 2013 [citado el 30 de diciembre del 2019]; 2013: 507034. Disponible en: https://doi.org/10.1155/2013/507034 
5. Chrisman NJ. The health seeking process: an approach to the natural history of illness. Cult Med Psychiatry [Internet]. 1977[citado el 14 de diciembre del 2019];1(4):351-77. Disponible en: https://doi. org/10.1007/BF00116243

6. Kleinman A. Concepts and a model for the comparison of medical systems as cultural systems. Soc Sci Med [Internet]. 1978 [citado el 10 de diciembre del 2019]; 12(2B):85-93. Disponible en: https://doi. org/10.1016/0160-7987(78)90014-5

7. Young A. The anthropologies of illness and sickness. Annu Rev Anthropol [Internet]. 1982 [citado el 20 de diciembre del 2019]; 11:257-85Disponible en: https://doi.org/10.1146/annurev. an.11.100182.001353

8. Schwartz LR. The hierarchy of resort in curative practices: The Admiralty Islands, Melanesia. J Health Soc Behav [Internet]. 1969 [citado el 14 de diciembre del 2019];10(3):201-9. Disponible en: https://doi. org/10.2307/2948390

9. Simionato I, Moraes M, Viera A, Seles L, Samboni T, Arrollo L, et al. Social determinants, their relationship with leprosy risk and temporal trends in a triborder region in Latin America. PLoS Negl Trop Dis [Internet]. 2018[citado el 7 de diciembre del 2019]; 12(4): e0006407. Disponible en:https://doi.org/10.1371/journal.pntd.0006407

10. Chamorro A, Tocornal C. Prácticas de salud en las comunidades del Salar de Atacama: Hacia una etnografía médica contemporánea. Estud Atacam [Internet]. 2005[citado el 10 de diciembre del 2019];(30):117-34. Disponible en: http://dx.doi.org/10.4067/S0718-10432005000200007

11. Honrado ER, Tallo V, Balis AC, Chan GP, Cho SN. Noncompliance with the world health organization-multidrug therapy among leprosy patients in Cebu, Philippines: its causes and implications on the leprosy control program. Dermatol Clin [Internet]. 2008 [citado el 17 de enero del 2020]; 26 (2): 221-9. Disponible en: https://doi.org/10.1016/j.det.2007.11.007

12. Heukelbach J, Chichava OA, Rodriguez de Oliveira A, Häfner K, Walther F Morais de Alencar CH, et al. Interruption and Defaulting of Multidrug Therapy against Leprosy: Population-Based Study in Brazil's Savannah Region. PLoS Negl Trop Dis [Internet]. 2011[citado el 30 de diciembre del 2019]; 5(5): e1031. Disponible en: https://doi.org/10.1371/journal.pntd.0001031

13. Good BJ. Medicina, racionalidad y experiencia: una perspectiva antropológica. Barcelona: Bellaterra Ediciones Barcelona; 2003.

14. Van Brakel WH, Sihombing B, Djarir H, Beise K, Kusumawardhani $\mathrm{L}$, Yulihane R, et al. Disability in people affected by leprosy: the role of impairment, activity, social participation, stigma and discrimination. Glob Acción para la Salud [Internet]. 2012[citado el 10 de diciembre del 2019]; 5:10. Disponible en: https://www.ncbi.nlm.nih.gov/pmc/articles/ PMC3402069

15. Naaz F, Mohanty PS, Bansal AK, Kumar D, Gupta UD. Challenges Beyond Elimination in Leprosy. Int J Mycobacteriol [Internet]. 2017[citado el 10 de diciembre del 2019]; 6(3):222-8. Disponible en: http://www.ijmyco. org/text.asp?2017/6/3/222/211929

16. Sermrittirong S, Van Brakel W, Kraipui N, Traithip S, Bunders-Aelen J. Comparing the perception of community members towards leprosy and tuberculosis stigmatization. Lepr Rev. 2015; 86(1): 54-61.
17. Valsa A, Longmore M, Ebenezer M, Richard J. Effectiveness of Social Skills Training for reduction of self-perceived Stigma in Leprosy Patients in rural India - a preliminary study. Lepr Rev. 2012; 83(1): 80 - 92.

18. Adhikari B, Kaehler N, Chapman RS, Raut S, Roche P. Factors Affecting Perceived Stigma in Leprosy Affected Persons in Western Nepal. PLoS Negl Trop Dis [Internet]. 2014[citado el 7 de diciembre del 2019]; 8(6): e2940. Disponible en: https://doi.org/10.1371/journal.pntd.0002940

19. Susanti IA, Mahardita NGP, Alfianto R, Sujana IMIWC, Siswoyo, Susanto T. Social stigma, adherence to medication and motivation for healing: A cross-sectional study of leprosy patients at Jember Public Health Center, Indonesia. J Taibah Univ Med Sci [Internet]. 2017[citado el 5 de diciembre del 2019]; 13 (1): 97-102. Disponible en: https://doi. org/10.1016/j.jtumed.2017.06.006

20. Li J, Yang L, Wang Y, Liu H, Cross H. How to improve early case detection in low endemic areas with pockets of leprosy: a study of newly detected leprosy patients in Guizhou Province, People's Republic of China. Lepr Rev. 2016; 87(1): 23-31.

21. Martins RJ, Oliveira ME, Saliba SA, Saliba SA, Ísper AJ. Sociodemographic and epidemiological profile of leprosy patients in an endemic region in Brazil. Rev Soc Bras Med Trop [Internet]. 2016[citado el 5 de enero del 2020]; 49(6):777-780. Disponible en: http://dx.doi.org/10.1590/00378682-0069-2016

22. Lira KB, Leite JJ, Maia DC, Freitas R, Feijão AR. Knowledge of the patients regarding leprosy and adherence to treatment. Braz J Infect Dis [Internet]. 2012 [citado el 11 de enero del 2020]; 16(5):472-5. Disponible en: http:// dx.doi.org/10.1016/j.bjid.2012.04.002

23. Matos AMF, Coelho ACO, Araújo LPT, Alves MJM, Baquero OS, Duthie MS, et al. Assessing epidemiology of leprosy and socio-economic distribution of cases. Epidemiol Infect [Internet]. 2018 [citado el 7 de diciembre del 2019]; 146(14): 1750-55. Disponible en: https://doi.org/10.1017/ S0950268818001814

24. Eysaguirre CF. El proceso de incorporación de la medicina tradicional y alternativa y complementaria en las políticas oficiales de salud [tesis de máster]. Lima: Facultad de ciencias sociales, Universidad Mayor de San Marcos; 2016.Disponible en: http://cybertesis.unmsm.edu.pe/handle/cybertesis/6274.

25. Cairns W, Smith S. Leprosy: making good progress but hidden challenges remain. Indian J Med Res [Internet]. 2013[citado el 11 de enero del 2020];137:1-3. Disponible en: https://www.ncbi.nlm.nih.gov/pmc/ articles/PMC3657869/

26. Zhang F, Chen S, Sun Y, Chu T. Healthcare seeking behaviour and delay in diagnosis of leprosy in a low endemic area of China. Lepr Rev [Internet]. 2009[citado el 11 de enero del 2020]; 80(4):416-23. Disponible en: https:/www.lepra.org.uk/platforms/lepra/files/lr/Dec09/ Lep416-423.pdf.

27. Walker SL, Withington SG, Lockwood DNJ. Leprosy. En: Farra J, Hotez P, Junghanss T, Kang G, Lalloo, White N, editores. Manson's Tropical Infectious Diseases[Internet]. 23a ed. 2014[citado el 12 de enero del 2020]. p 506-18 Disponible en: https://www.elsevier.com/books/mansons-tropical-infectious-diseases/9780702051012 